For reprint orders, please contact: reprints@futuremedicine.com

\title{
To what extent will 5-aminolevulinic acid change the face of malignant glioma surgery?
}

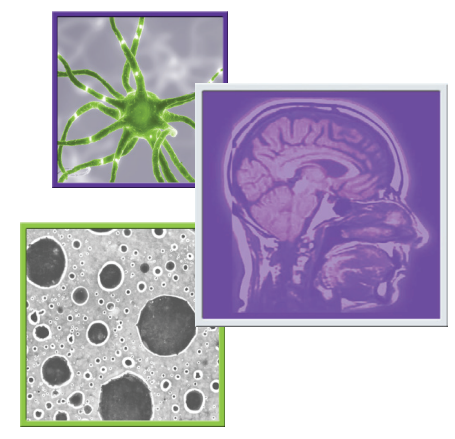

Ricardo Díez Valle ${ }^{*, 1} \&$ Sonia Tejada Solis ${ }^{1}$

\section{Practice points}

- High-grade glioma surgery aims to achieve complete resection of the contrast-enhancing tumor as observed on MRl; however, this result was achieved less than $30 \%$ of the time until quite recently.

- The use of fluorescence-guided surgery (FGS) with 5-aminolevulinic acid allows a substantial increase in resection, with complete resection of contrast-enhancing tumor possible in over $80 \%$ of patients.

- FGS is widely used in Europe and is likely to be the standard surgery for high-grade glioma in a few years.

- Complete resection of the enhancing tumor will become the typical result after surgery.

- Neurophysiological monitoring will be used more extensively.

- Trials will be stratified by resection outcome.

- The synergy between adjuvant therapy and complete resection will become more important.

- Distant or multifocal recurrence will become a more relevant problem in glioblastoma.

- Properly trained and dedicated oncological neurosurgeons are needed to obtain the full benefit of this technique.

- FGS with 5-aminolevulinic acid could be replaced by a better visualization system with the same consequences, although no such system is foreseeable now.

- An unexpected, disruptive new therapy that renders surgery unnecessary would change the expected scenario.

SUMMARY Glioma surgery is an essential part of glioma management; however, fully achieving the goal of surgery has been uncommon. The goal of surgery is 'maximal safe resection' with the accepted target for maximal being complete resection of the contrastenhancing tumor. This ideal result was obtained in less than $30 \%$ of cases in centers of excellence until a few years ago. The development of fluorescence-guided surgery using 5 -aminolevulinic acid has initiated a radical change. Over the past 5 years, various groups have published rates of complete resection of the enhancing tumor that exceed $80 \%$. In the coming years, as the use of the technology expands, complete resection should become a common, predictable result at many centers. Consequently, adjuvant therapies that benefit from resection could play a bigger role, resection could be incorporated as a variable in randomized trials and distant recurrence might become a more common problem.

\section{Background}

To apply the right perspective to fluorescence-guided surgery, the role of surgery in the management of malignant glioma must be briefly reviewed and understood. While the benefits of surgery

'Department of Neurosurgery, Clínica Universidad de Navarra, Navarre, Spain

*Author for correspondence: rdiezvalle@unav.es

\section{Future $\because$ Medicine $\%$ part of}




\section{KEYWORDS}

-5-aminolevulinic acid

- fluorescence-guided

surgery • glioblastoma

- malignant glioma • surgery for histological diagnosis and initial symptom alleviation have never been disputed, the benefit on overall survival (OS) has been a controversial subject for many years and still is to a certain degree. Malignant glioma are incurable tumors, and surgery is never fully total or radical because the tumors are diffuse and infiltrate the brain at a distance. The discussion involves the extent to which surgery, as well as radiotherapy and chemotherapy, can increase the median OS and the slim chance of long-term survival. As the oldest treatment, issues involving equipoise have precluded the benefits of studying surgery in large randomized trials, and these limits will probably never be surmounted.

Malignant glioma are a group of several entities; glioblastoma (GBM) is the most common and malignant of the group, so the data mentioned in this review are obtained mainly for GBM and assumed to be mostly valid for the other malignant gliomas, for which much less information is available. These tumors can be classified as having two components: a solid tumor and an infiltrative component [1]. The accepted target for surgery is the volume inside the contrast-enhancing areas, which is assumed to correspond grossly to the solid part.

When early postoperative MRI began to be used to assess the extent of resection (EOR), two things became clear. On the one hand, the neurosurgeon's impression of the resection was very imprecise; on the other hand, resection verified by MRI strongly correlated with a longer OS [2]. In a paper published by Albert et al., the neurosurgeons had estimated the resection to be completed in $54 \%$ of the cases, while MRI confirmed this finding in only $18 \%$ of the cases. This discrepancy highlighted the problem that the tumor tissue is difficult to discern in the surgical field with the naked eye or even the surgical microscope. Since that time, larger series $[3,4]$ that measured the extent of resection by MRI have consistently shown that patients with a higher volume resected have the longest mean OS, with nearly all long-term survivors falling into this category. The earlier literature, which failed to show an association between resection and OS, gauged resection only by the neurosurgeon's impression [5] and not with an objective, high-quality image control. A review of series that applied image quality control to the EOR shows that complete resection of enhancing tumor contrast-enhancing tumor (CRET) is achieved in a minority of cases, with rates less than $30 \%$ even at excellence centers in the world [6].

A more recent review by a group at the MD Anderson Cancer Center collected the largest series and most detailed analyses thus far, and it identified a continuous, nonlinear relationship between expected median OS and the EOR [7].

In the absence of randomized trials, it can be argued that the benefit of resection is unproven and could be attributed to an association with other factors, such as location and extent of the tumor. The best evidence is level $2 \mathrm{~b}$ from a trial by Stummer et al., in which the patients were randomized not to have better surgery but to have surgery with a tool that increases the EOR [8].

With these data, CRET has been considered beneficial for the patient but only possible in a minority of cases. EOR has not been measured properly at many centers and has been somewhat neglected by the neurooncology community; many large randomized trials of new therapies have not included an objective measurement of resection [9-11].

Interestingly, patients with complete resection have also been shown to benefit more from adjuvant chemotherapy [12].

\section{- Role of fluorescence-guided surgery}

Fluorescence-guided surgery (FGS) with 5-aminolevulinic acid (5-ALA) was introduced into the field of neurosurgery by Walter Stummer in the late nineties [13]. In this technique, the patient receives 5-ALA orally approximately $3 \mathrm{~h}$ before the induction of anesthesia. The synthesis of 5-ALA is the limiting step in the synthesis of a heme group; therefore, 5-ALA administration increases this metabolic pathway, leading to an increase in fluorescent molecules such as protoporphyrin IX (PpIX). Interestingly, glioma tumor cells accumulate much more PpIX than do normal cells. This difference is enough to be visible to the human eye and has been observed in all GBM cells lines and all GBM cases in most reported series. The molecular mechanisms underlying the accumulation are not perfectly known, and some metabolic anomalies have been reported, including high mRNA levels of coproporphyrinogen oxidase [14]. Because 5-ALA is unable to cross the intact blood-brain barrier, in vivo efficacy requires some degree of opening; this is not a problem for GBM treatment because the malignancy extensively disrupts the blood-brain barrier, but it most likely 
limits the efficacy in lower grade glioma cases, in which the fluorescence is usually absent in grade 2 and sometimes focal in grade 3 [15].

The surgical microscope was modified with filters to illuminate the field with a blue-violet light and maximize the detection of red fluorescence from PpIX [16]. The result is that the neurosurgeon is able to differentiate the tumor from normal tissue directly within the surgical field and in real time. The positive predictive value of the fluorescence for the presence of tumor is markedly high (well over 90\%) and has been validated by several groups [17-19]. In fact, 5-ALA surgery highlights not only the solid tumor, which appears deep red, but also a gradient of tumor cells invading the surrounding tissue, which presents a slowly fading intensity of fluorescence, giving the surgeons an estimation of the degree of tumor invasion that approaches that of histopathological study [17]. The negative predictive value is lower and depends on definition of tumor, as GBM is an infiltrative tumor in which isolated cells can be found far from the tumor to be resected; values of $26-50 \%$ have been reported [17-18,20].

A Phase III randomized trial was published in 2006 and showed a CRET rate of $65 \%$ for the use of 5-ALA versus 36\% for standard surgery [21]. This study was a multicenter trial conducted when the utility of the technique was still unproven, and the performance was irregular between centers [Stummer W, Unpublished Data]. It should be noted that 5-ALA is only a tool used to visualize; it does not have any effect on the tumor, so its performance depends on the individual surgeon. If the observed tumor is not resected, 5-ALA will not offer any benefit. After the randomized trial, better results have been reported by many other groups, with CRET rates of approximately $80 \%$ or more [22-24]. It should be remembered that the only role of 5-ALA is to increase CRET rate, any benefit in OS would be through resection; the previous randomized trial was not powered nor designed to study OS, where many variables come into play.

The use of 5-ALA has grown steadily from the original 19 centers participating in the randomized trial; as of October 2014, more than twenty thousand patients have been treated worldwide, and it is currently being used in 660 hospitals. There is a significant geographical asymmetry, as 400 of these hospitals are in Europe (one center per 1,700,000 inhabitants) and 135 are in Germany (one center per 592,000 inhabitants). The early trials and the regulatory trial were conducted in Germany, and the drug was approved by the European Regulator (EMA) in September 2007. While the rest of the world has gained access later, implementation has been particularly delayed in the USA, where it is not approved, although selected high-quality centers are using it in clinical trials. In Germany, each center with the system available would need to treat only 36 high-grade glioma cases a year to offer FGS to all patients in the country. Throughout Europe, all of the highgrade glioma cases would amount to 102 cases per year per center, or two per week, so there are already more than enough centers to offer the treatment to every patient.

Side effects for 5-ALA are mild, including a risk of sunburn if exposed to direct light during the first $24 \mathrm{~h}$ and an elevation of liver enzymes; no severe reactions have been described [21].

Considering the expansion of this treatment method in Germany and Europe, it should be expected that within the next $5-10$ years, most centers, and surely all centers with dedicated neurooncology programs in the developed world, will be using it.

The need to use a surgical microscope is an obstacle to adoption of the method, as not all neurosurgeons prefer to use the microscope for every case. In this regard, the possibility of using the system with other optical systems will help promote adoption. The system has already been shown to work with surgical endoscopes [25,26], exoscopes [27] and surgical loupes [28]. The data available for these other systems are not as complete as the data for microscopes, so they need to be verified carefully, especially for electronic display systems, to avoid the risk of image overamplification causing false-positive images. However, the optical principles are the same, and the system should work if the appropriate parameters for light, filtering and image display are carefully worked out and histopathological validation is performed.

\section{- Consequences of widespread use of FGS}

The generalized use of FGS will lead to a fundamental change in malignant glioma surgery. Over the next 5-10 years, FGS with 5-ALA will become the standard surgery, and many more centers will achieve complete resection in a majority of cases rather than only occasionally. This change can be expressed as a higher 
percentage of patients achieving complete resection, but it will also mean that a very high percentage of patients will have very little residual tumor [24].

Importantly, in addition to the increase in resection, surgery for malignant glioma will have a much more predictable result. As has been described before, the benefit of resection is accepted by a majority of researchers, but it is considered to be an occasional outcome and is therefore not included in most trials as a variable. When CRET becomes the typical result in considerably more than half of patients, the benefit of adjuvant therapy will be considered differently for resected cases versus nonresected cases.

Therapies that benefit more from resection will be more useful. This assumption has already been found to be relevant for certain types of immunotherapy [29,30].

Another important consequence is that, as the local problem is better solved, the survival will improve by months, but more distant recurrences will then appear [31]. Currently, over $80 \%$ of patients with GBM have a local recurrence, and almost all research on tumor biology is performed on the main tumor mass; very little effort is directed toward the invasive cells that are distant to the mass and will be responsible for the late recurrences, albeit some studies have shown that the invasive cells are different $[32,33]$. With more extensive resections, the local problem should be better controlled, and the study of invasive disease will become more widespread.

At the same time, the unique ability of FGS to differentiate in vivo solid tumors from infiltration areas allows selective sampling of the peripheral areas. In this way, the interface between tumor and normal parenchyma can be studied, and invasive cells can be isolated [17]. Some papers have shown already that tumor initiating cells from the periphery are different from tumor initiating cells from the central mass $[34,35]$. This finding means that therapies designed against the central portion, which currently describes nearly all targeted therapies, are not equally efficient at preventing distant recurrence. As FGS becomes more widespread, more research groups will gain access to this selective sampled tissue, and a better understanding of this invasive disease is expected.

Using FGS, the tumor is clearly visible in the surgical field; therefore, the only reason to leave tumor tissue behind should be that the tumor affects the so-called eloquent areas of the brain, areas whose lesion could produce a neurological deficit for the patient. Minor exceptions to this general rule happen sometimes with small parts of the tumor going undetected due to an irregular or deep surgical field or the existence of small satellite nodules. Because FGS is a surface-viewing technique, it will not detect anything that is covered by normal tissue or not properly exposed. It has been proposed that if this happens, the patient can be reoperated a few days later to obtain CRET without significant additional risks [36].

When a tumor invades functional areas of the brain, the resection may need to be limited to avoid significant deficits. In this situation, resecting all of the fluorescent tissue could produce a neurological deficit. FGS is helpful to differentiate solid tumor, which is not functional tissue and can be resected, from invasive areas, or normal tissue. The appropriate way to deal with tumors near eloquent regions is to use neurophysiological monitoring intraoperatively, including awake surgery if language needs to be monitored. Neurophysiological monitoring is a well-established technique, and its combination with FGS has already been reported [23,37]; these two groups have shown that this combination can result in markedly high rates of resection with minimal morbidity, even in eloquent locations. However, neurophysiological monitoring adds to the complexity of surgery and is not available everywhere. Today, incomplete resection is still considered to be quite common and acceptable; therefore, in many centers that do not have routine neurophysiological monitoring available, a portion of the tumor is left in doubtful cases to avoid risk. In the near future, CRET or resection limited by neurophysiological monitoring should become the rule rather than the exception, and the level of treatment expected from the neurosurgeon and the institution will be better.

\section{- Alternative technologies}

There are other options leading the transition toward more complete resections, similar to FGS, and a short review is provided here.

Intraoperative MRI (iMRI) has seen a large expansion over the past 10 years. The main use of this equipment is to guide the resection of glioma before the end of surgery and to continue the resection until the objective is achieved. Very high rates of CRET have been reported with the routine use of iMRI [38], with increased patient 
survival and a wider EOR [39]; these benefits have been demonstrated in a randomized trial [40]. The final objective is similar to that of FGS, although there are important differences:

- iMRI control is useful for any type of glioma, whereas FGS is only applicable to malignant glioma because most low-grade tumors do not fluoresce [41];

- FGS is already available to a majority of patients in Europe and could be available to most of the developed world within the next few years. iMRI has been installed in selected centers in the developed world; however, the cost of installation and maintenance of an iMRI scanner make its use much more limited than that of FGS. It will not be available to most patients in the foreseeable future, unless a rather significant change in cost occurs;

- FGS provides real-time, direct visual guidance. Resection can be adjusted to portions of the tumor of a millimeter or less, with knowledge of the approximate tumor cell density of the tissue able to be applied while it is being resected. iMRI offers a quality control of the work performed; to use it, the surgery must be stopped for approximately $45 \mathrm{~min}$, and if the resection is not sufficient, the surgery can be started again, but it does not offer real-time control. This method does not allow a correction if nontumor tissue is resected, and due to time limits, performing it more than two- or three-times during a surgery is impractical;

- MRI is useful for the control of CRET, whereas 5-ALA staining goes farther. A complete resection of the fluorescent tissue indicated by 5-ALA takes out more tumor than CRET alone [42]. A series has been published in which patients with CRET and complete resection of the fluorescent tissue lived longer than did patients with CRET but some residual fluorescence [43].

Other fluorescence systems:

- The utility of 5-ALA has led to further investigation of other uses of fluorescence, although none has currently shown an equivalent efficacy in clinical use. Sodium fluorescein (SF) has been used in the past for the same indication; it was used as early as 1948 [44] and then restudied in the era of modern surgery $[45,46]$, but it achieved little success due to its lack of specificity for tumor tissue. The fundamental difference is that SF itself fluoresces; it can therefore stain vessels and normal tissue and is not exclusive to tumor cells. Still, some groups have studied it again after the 5-ALA success, using a specially designed filter in the microscope $[47,48]$. No detailed histopathological correlation has been published equivalent to that of 5-ALA, and even with the improved filter, it is difficult to accept that it might be equivalent to 5-ALA for high-grade glioma, given its unspecific mechanism of action. The main advantage reported is the price, but that factor does not take into consideration the fact that SF for glioma is an off-label indication.

Many other technologies like ultrasound, functional MRI and tractography can be helpful for the resection of glioma, but are more complementary than alternatives to FGS, and a detailed comparison to those techniques is outside of the scope of the manuscript.

\section{- Challenges}

FGS is only a tool to help visualize the tumor, not a drug for treating the tumor. To make the most of this help, neurosurgeons need the appropriate skill, training, dedication and patience; this type of surgery takes longer. Neurosurgeons who have little experience with parenchymal tumors and neurosurgeons who remain unconvinced of the benefits of surgery will not achieve improved resection.

Improvements in the treatment of high-grade glioma can come in many ways. As long as the improvements to new therapies are complementary with resection, which has been the case until now, this vision will hold true. Disruptive improvements could appear that enable treatment of the disease even with a major tumor burden, so surgery could be made obsolete. Despite all of the new knowledge of the molecular biology of the tumor, this silver bullet does not seem to be any nearer now than it has been in the past.

FGS with 5-ALA will be displaced only if a superior technology appears that has the same or better selectivity for tumor tissue and the same or better ease of use. In this case, the tool would be replaced, but the consequences for malignant glioma surgery would be the same.

\section{- Cost considerations}

Of course, cost is now an important issue for any medical advance. 5-ALA costs approximately 
1000 euros per patient (note that this cost can be affected by factors such as the patients weight, and this is not intended to be a detailed cost/ efficacy study), and the surgical microscopes needed to execute it include only the top models of the major manufacturers, with costs of approximately 300,000 euros. Very grossly, the surgical microscope can be used for 10 years in the treatment of over 200 patients per year, making the per-patient cost approximately 150 euros per case. Although these are significant costs, they are only a fraction of the total cost of a craniotomy for a tumor. FGS has been shown to be cost-efficient in a pharmaco-economic review in Spain [49]. The drug is even more cost-efficient if it is compared not only to the cost of standard craniotomy but to the total cost of therapy for malignant glioma, including radiotherapy and chemotherapy.

In the future, the cost of the drug should become cheaper as the exclusive rights of the patent expire.

The option of using the system without a microscope could also help its adoption in countries with fewer economic resources, as endoscopes, exoscopes and surgical loupes are more widely available than are surgical microscopes [25-28].

\section{Conclusion \& future perspective}

As the use of FGS extends, surgery for high grade glioma is undergoing a transition from unpredictable results with few complete resections to a majority of complete resections. The tolerance for unnecessarily limited surgery should become narrower. Expertise and skill of neurosurgeons and the use of neurophysiological monitoring are needed to get the full benefit of the technique, so in the authors' perspective, it will become more common practice in neurooncology centers.

Any degree of resection is insufficient therapy for malignant glioma, but the benefit is synergistic with other adjuvant therapies, so the change in the resection model will lead to trials stratified by resection, and to a greater benefit from some therapies. A better local control will increase median OS by some months, to be followed by more distant recurrences, and by an increase in the effort directed to understanding the distant, invasive component of the tumor.

At present, there is no better way to visualize tumors than 5-ALA, and if such a technique would be developed, the consequences will likely be the same with the alternative system. Only a disruptive totally new therapy that could make surgery unnecessary would change the expected scenario.

Financial \& competing interests disclosure

$R$ Diez Valle has received speaker fees from Medac $G m b H$, Wedell, Germany. The authors have no other relevant affiliations or financial involvement with any organization or entity with a financial interest in or financial conflict with the subject matter or materials discussed in the manuscript apart from those disclosed.

No writing assistance was utilized in the production of this manuscript.

\section{References}

1 Kelly PJ, Daumas-Duport C, Kispert DB, Kall BA, Scheithauer BW, Illig JJ. Imagingbased stereotaxic serial biopsies in untreated intracranial glial neoplasms. J. Neurosurg. 66(6), 865-874 (1987).

2 Albert FKMD, Forsting MMD, Sartor KMD, Adams HDIM, Kunze SMD. Early postoperative magnetic resonance imaging after resection of malignant glioma: objective evaluation of residual tumor and its influence on regrowth and prognosis.

Neurosurgery 34(1), 45-61 (1994).

3 Lacroix M, Abi-Said D, Fourney DR et al. A multivariate analysis of 416 patients with glioblastoma multiforme: prognosis, extent of resection, and survival. J. Neurosurg. 95(2), 190-198 (2001).

4 Sanai N, Polley MY, McDermott MW, Parsa AT, Berger MS. An extent of resection threshold for newly diagnosed glioblastomas. J. Neurosurg. 115(1), 3-8 (2011).

5 Nazzaro JM, Neuwelt EA. The role of surgery in the management of supratentorial intermediate and high-grade astrocytomas in adults. J. Neurosurg. 73(3), 331-344 (1990).

6 Sanai N, Berger MS. Glioma extent of resection and its impact on patient outcome. Neurosurgery 62(4), 753-64; discussion 264-6 (2008).

7 Marko NF, Weil RJ, Schroeder JL, Lang FF, Suki D, Sawaya RE. Extent of resection of glioblastoma revisited: personalized survival modeling facilitates more accurate survival prediction and supports a maximum-saferesection approach to surgery. J. Clin. Oncol. 32(8), 774-782 (2014).

8 Stummer W, Reulen HJ, Meinel T et al. Extent of resection and survival in glioblastoma multiforme: identification of and adjustment for bias. Neurosurgery 62(3), 564-76; discussion 564-76 (2008).

9 Stupp R, Mason WP, van den Bent MJ et al. Radiotherapy plus concomitant and adjuvant temozolomide for glioblastoma. N. Engl. J. Med. 352(10), 987-996 (2005).

10 Chinot OL, Wick W, Mason W et al. Bevacizumab plus radiotherapy-temozolomide for newly diagnosed glioblastoma. N. Engl. J. Med. 370(8), 709-722 (2014).

11 Gilbert MR, Dignam JJ, Armstrong TS et al. A randomized trial of bevacizumab for newly diagnosed glioblastoma. N. Engl. J. Med. 370 (8), 699-708 (2014).

12 Stummer W, van den Bent MJ, Westphal M. Cytoreductive surgery of glioblastoma as the key to successful adjuvant therapies: new arguments in an old discussion. Acta Neurochir. (Wien) 153(6), 1211-1218 (2011). 
13 Stummer W, Stocker S, Wagner S et al. Intraoperative detection of malignant gliomas by 5 -aminolevulinic acid-induced porphyrin fluorescence. Neurosurgery 42(3), 518-525; discussion 525-6 (1998).

14 Takahashi K, Ikeda N, Nonoguchi N et al. Enhanced expression of coproporphyrinogen oxidase in malignant brain tumors: CPOX expression and 5-ALA-induced fluorescence. Neuro Oncol. 13(11), 1234-1243 (2011).

15 Widhalm G, Wolfsberger S, Minchev G et al. 5-Aminolevulinic acid is a promising marker for detection of anaplastic foci in diffusely infiltrating gliomas with nonsignificant contrast enhancement. Cancer 116(6), 1545-1552 (2010).

16 Stummer W, Stepp H, Moller G, Ehrhardt A, Leonhard M, Reulen HJ. Technical principles for protoporphyrin-IX-fluorescence guided microsurgical resection of malignant glioma tissue. Acta Neurochir. (Wien) 140(10), 995-1000 (1998).

17 Idoate MA, Diez Valle R, Echeveste J, Tejada S. Pathological characterization of the glioblastoma border as shown during surgery using 5-aminolevulinic acid-induced fluorescence. Neuropathology 31(6), 575-582 (2011).

18 Roberts DW, Valdes PA, Harris BT et al. Coregistered fluorescence-enhanced tumor resection of malignant glioma: relationships between delta-aminolevulinic acid-induced protoporphyrin IX fluorescence, magnetic resonance imaging enhancement, and neuropathological parameters. Clinical article. J. Neurosurg. 114(3), 595-603 (2011).

19 Stummer W, Tonn JC, Goetz C et al. 5-Aminolevulinic acid-derived tumor fluorescence: the diagnostic accuracy of visible fluorescence qualities as corroborated by spectrometry and histology and postoperative imaging. Neurosurgery 74 (3), 310-319; discussion 319-320 (2014).

20 Stummer W, Novotny A, Stepp H, Goetz C, Bise K, Reulen HJ. Fluorescence-guided resection of glioblastoma multiforme by using 5-aminolevulinic acid-induced porphyrins: a prospective study in 52 consecutive patients. J. Neurosurg. 93(6), 1003-1013 (2000).

21 Stummer W, Pichlmeier U, Meinel T et al. Fluorescence-guided surgery with 5-aminolevulinic acid for resection of malignant glioma: a randomised controlled multicentre Phase III trial. Lancet Oncol. 7(5), 392-401 (2006).

22 Della Puppa A, Ciccarino P, Lombardi G, Rolma G, Cecchin D, Rossetto M. 5-Aminolevulinic acid fluorescence in high grade glioma surgery: surgical outcome, intraoperative findings, and fluorescence patterns. Biomed. Res. Int. 2014, 232561 (2014).

Schucht P, Beck J, Abu-Isa J et al. Gross total resection rates in contemporary glioblastoma surgery: results of an institutional protocol combining 5-aminolevulinic acid intraoperative fluorescence imaging and brain mapping. Neurosurgery 71(5), 927-936 (2012).

24 Diez Valle R, Tejada Solis S, Idoate Gastearena MA, Garcia de Eulate R, Dominguez Echavarri P, Aristu Mendiroz J. Surgery guided by 5 -aminolevulinic fluorescence in glioblastoma: volumetric analysis of extent of resection in single-center experience. J. Neurooncol. 102(1), 105-113 (2011).

25 Rapp M, Kamp M, Steiger HJ, Sabel M. Endoscopic-assisted visualization of 5 -aminolevulinic acid-induced fluorescence in malignant glioma surgery: a technical note. World Neurosurg. 82(1-2), e277-e279 (2014).

26 Schwartz TH. Endoscopic-assisted 5 -aminolevulinic acid imaging. World Neurosurg. 82(1-2), e117-e118 (2014).

27 Belloch JP, Rovira V, Llacer JL, Riesgo PA, Cremades A. Fluorescence-guided surgery in high grade gliomas using an exoscope system. Acta Neurochir. (Wien) 156(4), 653-660 (2014).

28 Kuroiwa T, Kajimoto Y, Furuse M, Miyatake S. A surgical loupe system for observing protoporphyrin IX fluorescence in high-grade gliomas after administering 5 -aminolevulinic acid. Photodiagnosis Photodyn. Ther. 10(4), 379-381 (2013).

29 Liau LM, Prins RM, Kiertscher SM et al. Dendritic cell vaccination in glioblastoma patients induces systemic and intracranial T-cell responses modulated by the local central nervous system tumor microenvironment. Clin. Cancer Res. 11(15), 5515-5525 (2005).

30 Sampson JH, Heimberger AB, Archer GE et al. Immunologic escape after prolonged progression-free survival with epidermal growth factor receptor variant III peptide vaccination in patients with newly diagnosed glioblastoma. J. Clin. Oncol. 28(31), 4722-4729 (2010).

31 Tejada S, Aldave G, Marigil M, Gallego Perez-Larraya J, Dominguez PD, Diez-Valle R. Factors associated with a higher rate of distant failure after primary treatment for glioblastoma. J. Neurooncol. 116(1), 169-175 (2014).

32 Giese A, Bjerkvig R, Berens ME, Westphal $M$. Cost of migration: invasion of malignant gliomas and implications for treatment. J. Clin. Oncol. 21(8), 1624-1636 (2003).

33 Hoelzinger DB, Mariani L, Weis J et al. Gene expression profile of glioblastoma multiforme invasive phenotype points to new therapeutic targets. Neoplasia 7(1), 7-16 (2005).

34 Piccirillo SG, Dietz S, Madhu B et al. Fluorescence-guided surgical sampling of glioblastoma identifies phenotypically distinct tumour-initiating cell populations in the tumour mass and margin. Br. J. Cancer 107(3), 462-468 (2012).

35 Rampazzo E, Della Puppa A, Frasson C et al. Phenotypic and functional characterization of Glioblastoma cancer stem cells identified trough 5-aminolevulinic acid-assisted surgery. J. Neurooncol. 116(3), 505-513 (2014).

36 Schucht P, Murek M, Jilch A et al. Early re-do surgery for glioblastoma is a feasible and safe strategy to achieve complete resection of enhancing tumor. PLoS ONE 8(11), e79846 (2013).

37 Della Puppa A, De Pellegrin S, d'Avella E et al. 5-aminolevulinic acid (5-ALA) fluorescence guided surgery of high-grade gliomas in eloquent areas assisted by functional mapping. Our experience and review of the literature. Acta Neurochir. (Wien) 155(6), 965-972 (2013).

38 Hatiboglu MA, Weinberg JS, Suki D et al. Impact of intraoperative high-field magnetic resonance imaging guidance on glioma surgery: a prospective volumetric analysis. Neurosurgery 64(6), 1073-1081; discussion 1081 (2009).

39 Kuhnt D, Becker A, Ganslandt O, Bauer M, Buchfelder M, Nimsky C. Correlation of the extent of tumor volume resection and patient survival in surgery of glioblastoma multiforme with high-field intraoperative MRI guidance. Neuro Oncol. 13(12) 1339-1348 (2011).

40 Senft C, Bink A, Franz K, Vatter H, Gasser T, Seifert V. Intraoperative MRI guidance and extent of resection in glioma surgery: a randomised, controlled trial. Lancet Oncol. 12(11), 997-1003 (2011).

41 Widhalm G, Kiesel B, Woehrer A et al. 5-Aminolevulinic acid induced fluorescence is a powerful intraoperative marker for precise histopathological grading of gliomas with non-significant contrast-enhancement. PLoS ONE 8(10), e76988 (2013).

42 Schucht P, Knittel S, Slotboom J et al. 5-ALA complete resections go beyond MR contrast enhancement: shift corrected volumetric analysis of the extent of resection in surgery for glioblastoma. Acta Neurochir. (Wien) 156(2), 305-312; discussion 312 (2014). 
43 Aldave G, Tejada S, Pay E et al. Prognostic value of residual fluorescent tissue in glioblastoma patients after gross total resection in 5-aminolevulinic acid-guided surgery. Neurosurgery 72(6), 915-920; discussion 920-921 (2013).

44 Moore GE, Peyton WT. The clinical use of fluorescein in neurosurgery; the localization of brain tumors. J. Neurosurg. 5(4), 392-398 (1948).

45 Kuroiwa T, Kajimoto Y, Ohta T. Development of a fluorescein operative microscope for use during malignant glioma surgery: a technical note and preliminary report. Surg. Neurol. 50 (1), 41-48; discussion 48-49 (1998).

46 Shinoda J, Yano H, Yoshimura S et al. Fluorescence-guided resection of glioblastoma multiforme by using high-dose fluorescein sodium. Technical note. J. Neurosurg. 99(3), 597-603 (2003).

47 Acerbi F, Broggi M, Eoli M et al. Fluoresceinguided surgery for grade IV gliomas with a dedicated filter on the surgical microscope: preliminary results in 12 cases. Acta Neurochir. (Wien) 155(7), 1277-1286 (2013).

48 Rey-Dios R, Cohen-Gadol AA. Technical principles and neurosurgical applications of fluorescein fluorescence using a microscopeintegrated fluorescence module. Acta Neurochir. (Wien) 155(4), 701-706 (2013).

49 Slof J, Diez Valle R, Galvan J. Costeffectiveness of 5-aminolevulinic acidinduced fluorescence in malignant glioma surgery. Neurologia 30 (3), 163-168 (2014). 